\title{
LA EXPERIENCIA MUSICAL DE LOS IMPROVISADORES DE LA ZONA ANDINA Y EL CARIBE COLOMBIANO
}

THE MUSICAL EXPERIENCE OF THE IMPROVISORS OF THE ANDEAN AREA AND THE COLOMBIAN CARIBBEAN

Henry Gustavo Roa Ordoñez *

Correo electrónico: henryroa54@gmail.com

Fabián Manuel Rojas Parra **

Correo electrónico: fabianm.rojas@correo.usa.edu.co

Fecha recibido: 31 - 07- 2019

Fecha de aprobación: 26 - 11 - 2019

* Realizó sus estudios musicales en el Conservatorio de la Universidad Nacional de Colombia, donde obtuvo el título de Licenciado en Pedagogía Musical, además es Magister en Evaluación en Educación de la Universidad Santo Tomás. Como docente investigador su labor se ha enfocado al desarrollo y la implementación de propuestas en el campo de la musicología, la educación musical y evaluación de programas académicos.
** Músico pianista con énfasis en pedagogía de la Universidad Nacional de Colombia y Magister en Pedagogía del piano de la misma institución. Se ha desempeñado como docente de piano y pianista correpetidor en varias universidades colombianas. En la Universidad Sergio Arboleda actualmente se dedica a la investigación en temas relacionados con la improvisación. 
Resumen

En el medio musical es ampliamente reconocida la importancia de la improvisación como recurso para el fomento del pensamiento activo y creativo del músico. Su práctica implica, entre otros aspectos: tomar decisiones en tiempo real, escuchar a otros, predecir y experimentar nuevas estructuras y organizaciones musicales, y llevar a cabo ajustes sensoriales y perceptivos necesarios para una adecuada interpretación. Este artículo sintetiza las principales concepciones y mecanismos desarrollados por una muestra significativa de improvisadores colombianos en sus respetivos contextos. Para ello, empleando una metodología etnomusicológica centrada en el estudio de caso, los autores examinan cómo estos músicos conciben la improvisación a partir de sus experiencias de vida. La investigación determina cómo la improvisación instrumental observada en los aires tradicionales se concreta gracias a una preparación previa dentro de cada marco cultural y por el concurso de algunos materiales y procedimientos concretos.

Palabras clave: Improvisación; preparación; música colombiana; costa Caribe; música andina.

\section{Abstract}

In the music world, the importance of improvisation as a means to promote the active and creative thinking of the musician is widely recognized. Its practice involves, among other things: making decisions in real time, listening to others, predicting and experimenting with new structures and musical organizations and carrying out sensory and perceptual adjustments necessary for an adequate interpretation. This article synthesizes the main conceptions and mechanisms developed by a representative sample of Colombian improvisers in their respective contexts. For this, using an ethnomusicological methodology centered on case study, the authors examine how these musicians conceive improvisation based on their life experiences. The study shows how the instrumental improvisation observed in traditional repertoire is carried out thanks to a previous training within each cultural framework and by the usage of some raw materials and procedures.

Keywords: Improvisation, preparation, Colombian music, Caribbean coast, Andean music. 


\section{Introducción}

Tradicionalmente, se ha considerado a la improvisación como una ejecución musical bajo la idea de lo imprevisto y de lo espontáneo y que, además, es propia de músicos excepcionalmente talentosos. Son inagotables sus definiciones, así como las múltiples miradas bajo las que suele ser estudiada y las distintas evocaciones que ella sugiere en el imaginario musical. Aseguran Nettl y Russel (2004), citando a Bailey (1992), que "de todas las actividades musicales, la improvisación goza de la curiosa distinción de ser la que más se practica y la que menos se reconoce y comprende” (p. 12). Muy seguramente, esta apreciación surge al considerar que la improvisación brota de forma repentina e imprevista, o porque hace parte de un estadio primario y necesario dentro de toda composición.

A pesar de las muchas discusiones acerca de los distintos factores que determinan su desarrollo, sobre los puntos de partida, metodologías y recursos, las influencias, el contexto natural en que se encuentra el músico, entre otros; la improvisación continúa ocupando un lugar secundario, igual que también son modestos los escritos y las referencias investigativas con respecto a las formas de hacer música. Señalan Nettl y Russel (2004) que en ciertos ámbitos de la música culta la improvisación es considerada como una práctica que carece de planificación, formalidad y previsión, mientras que para Gainza (2002) se trata de un evento musical que pone en acción la originalidad y la capacidad de exploración y creatividad de un músico.

Al respecto de la música académica, es importante observar cómo, con la llegada de instituciones y conservatorios en particular, la improvisación dejó de formar parte de los recursos creativos de los instrumentistas. En estos centros educativos la idea de conservar las tradiciones vino de la mano con la especialización del oficio musical. Desde ese momento, los instrumentistas dedicarían su tarea formativa únicamente a perfeccionar sus habilidades de ejecución y a la ampliación y conocimiento de repertorios, mientras que los compositores serían los únicos depositarios de la labor de creación musical. Sin embargo, no hay que olvidar que, muchas veces, las grandes obras del repertorio académico han surgido a partir de las improvisaciones que, por elaboración posterior, el compositor dota de gran complejidad. A menudo resulta extraño para un músico, formado bajo parámetros disciplinares alrededor de la lectura musical, encontrar a otros músicos que, casi sin pensarlo, realizan discursos musicales de manera espontánea y original. Asimismo, se olvida que "las actividades de improvisación pueden complementar y apoyar el estudio de la armonía escrita, habilidades auditivas, e incluso el análisis" (Rabinovitch \& Slominski, 2015, p. 27).

Justamente, uno de los tantos problemas que enfrenta el desarrollo de la improvisación está referido a la lectura musical. Por su carácter efímero ha estado históricamente enfrentada a la tradición escrita que domina las formas de enseñanza musical formal. Asegura Dalhaus (1992) que "la existencia misma de una historia de la música, y no solamente de una tradición, se debe a la escritura, que le ha permitido pasar del estado 
de la improvisación a aquel de la composición" (p. 86). Hasta hace poco, la música académica había desdeñado el ejercicio de la improvisación por considerarla como un arte incompleto en la medida en que es incapaz de sobrevivir por sí misma. Bailey (1992) afirma al respecto:

Además, existe una inequívoca sospecha de que la adquisición de capacidades de lectura tiene en cierta manera un efecto directo sobre las mermas de las capacidades de improvisación, y una aceptación de que, a menudo, estas dos cosas, no marchan bien juntas (citado por Sans, s.f., p. 10).

Bailey (1992), autor prestigioso en el campo de la improvisación, sostiene también que "la improvisación es un modo de acción, o incluso una actitud, que implica no sólo grados de espontaneidad sino también una valoración implícita o explícita de tal libertad con respeto a la actividad" (p. ix). Desde este punto de vista, la improvisación no será la misma en el momento de su transcripción y análisis porque su interpretación siempre será distinta y muy lejana de la versión que le dio origen. Por su carácter único es obligada a resolver sus propios desafíos creativos y cuenta para ello con sus propios medios y su propio saber hacer, en el que es evidente que las producciones no obedecen a hechos o interpretaciones estrictamente mecánicos.

Dentro del ámbito de la música colombiana no es frecuente hablar de la improvisación en la relación teórico-práctica, es decir, de las bases conceptuales de sus fundamentos, del porqué y para qué de sus acciones. Por el contrario, la educación formal en música tradicional colombiana suele ser abordada de la misma manera que el repertorio 'clásico'. A saber, centrada en el montaje de repertorios, dejando de lado una considerable parte de sus características de espontaneidad creativa.

En Colombia llevamos, en lo que al estudio de la música improvisada se refiere, un importante retraso, sin embargo, hay honrosas excepciones de aquellos que llevan años en el estudio de la improvisación como una práctica enriquecedora y con alto sentido musical para quienes la ejercen de forma activa. Aunque prevalece la escasez de fuentes al respecto, es posible partir de algunos antecedentes como el documento "Aplicación de conceptos de improvisación en el pasillo y el bambuco de la región Andina Colombiana”, publicado en el 2006 por Montalvo y Pérez. El trabajo es probablemente el primer esfuerzo reconocido en Colombia por establecer un camino de acción en el aprendizaje de la improvisación en ritmos colombianos. Si bien su objetivo puede parecer equivalente al que guía esta investigación, hay que precisar que, a diferencia de dichos autores, quienes intentan aportar nuevo conocimiento a la tradición a partir de la improvisación jazzística, este estudio se rige por la premisa de que la improvisación siempre ha formado parte de la tradición musical colombiana.

Lo anterior es más evidente en algunas zonas del país donde la improvisación es indispensable en cualquier realización musical. El porro y fandango, de las bandas tradicionales del departamento de Córdoba, son analizados en la tesis de maestría "La improvisación en el bombardino a partir del repertorio tradicional de las bandas del departamento de Córdoba", del año 2011, de Pacheco y Caraballo en la Universidad 
de Montería. Los autores develan las principales características de la improvisación realizada por los instrumentistas, con el objeto de organizar los elementos musicales en un banco de datos que se podría aprovechar posteriormente en la enseñanza de estos géneros.

En el presente trabajo investigativo se hace referencia al concepto de preparación para la improvisación, aludiendo al proceso previo que el improvisador desarrolla con el fin de producir un discurso coherente dentro de un estilo dado. Nos hemos propuesto, por un lado, estudiar las herramientas que utiliza a la hora de improvisar un grupo sobresaliente y representativo de músicos colombianos que han descollado por este tipo de habilidad y, por el otro, considerar la génesis y concepciones prácticas que ellos han elaborado para consolidar sus habilidades de improvisación.

Hay evidencias de que el improvisador posee fórmulas y recursos que prepara con anticipación, que lo capacitan para introducir cambios in situ al momento de improvisar. Indiscutiblemente, debe existir un claro dominio del instrumento y del lenguaje musical que le permitan un amplio margen para improvisar. Como lo expresan Nettl y Russell (2004), los improvisadores siempre cuentan con un material inicial:

Podemos afirmar, sin lugar a dudas, que los improvisadores siempre tienen un punto de partida, algo sobre lo cual improvisan. Existen numerosos puntos de partida: desde temas, melodías y secuencia de acordes hasta formas diversas, desde un repertorio de técnicas hasta un repertorio de motivos y materiales más extensos, desde los fácil y natural hasta lo intelectualmente complejo (p. 22).

Es indiscutible que el ejercicio de la improvisación responde a experiencias particulares ajenas; muchas de ellas, a procesos formativos sistemáticos dentro de los planes de estudios musicales, razón por la cual se hace necesario develar, desde estas referencias particulares, los diferentes procesos inmersos en el desarrollo de habilidades y visualizar, en el mediano plazo, posibles aplicaciones dentro del campo educativo musical.

Paulatinamente, las concepciones acerca de la improvisación han venido cambiando favorablemente. Si en épocas anteriores era considerada, con cierto desdén, como una manifestación propia de las expresiones tradicionales y populares llevada a cabo por músicos con escasa formación de tipo académico, hoy en día es vista como una herramienta, no solo para el fomento del pensamiento musical, sino como un recurso invaluable para recrear las prácticas tradicionales en los acostumbrados escenarios musicales.

El propósito de este estudio se encuentra en la necesidad de observar de manera analítica el modo en que distintas estrategias se manifiestan en consonancia con ritmos de la música tradicional colombiana en la región andina y la costa Caribe. De esta manera, la improvisación -como recurso formativo- podrá ganar más terreno, no para adoptar formas específicas, porque de ser así perdería su esencia, sino para contar con un espacio propio que permita comprender lo que los músicos improvisadores realmente hacen. 


\section{Marco teórico}

Las concepciones y definiciones acerca de la improvisación son diversas y amplias dependiendo de los contextos culturales, científicos y académicos en que se encuentre. Desde el punto de vista cultural, la improvisación posee diferentes connotaciones. Por ejemplo, para culturas orientales, la improvisación ocupa un papel preponderante con fuerte arraigo en la tradición oral y, por supuesto, con sus propios márgenes de libertad.

A pesar de las diferentes posturas y estudios, los cuestionamientos acerca de la improvisación continúan por cuanto se trata de un hecho creativo que surge desde la espontaneidad y cuál es en realidad el margen de preparación con el que cuenta. Al respecto, asegura Gainza (2002) que la improvisación abarca desde la libertad total hasta la sujeción a pautas o reglas estrictas, propias o ajenas al improvisador. Asimismo, sus rangos van desde el juego espontáneo irreflexivo hasta contar con los más altos niveles de participación de la conciencia.

Del mismo modo, debe mencionarse la 'improvisación libre', donde el hecho creativo se manifiesta sin la presencia de referentes a géneros o materiales previamente concebidos en cualquier contexto musical. En este caso, lo que importa es el juicio y el valor que cada músico le otorga a la exploración para reflejar sus propios gustos estéticos. Por supuesto, en este ámbito la improvisación no requiere de preparación, más aún cuando es empleada con fines educativos o terapéuticos.

Desde las perspectivas que rigen las ciencias de la cognición, Pressing $(1984 ; 1988)$ aborda el fenómeno de la improvisación como un mecanismo de realimentación constante que pone en juego las ideas y las habilidades creativas del improvisador a través de un sistema dinámico de procesamiento interior y de exteriorización continuo. La base de dicho proceso radica no solo en la percepción, sino que debe contar también con una memoria de base construida por experiencias anteriores y una de tipo kinésico, además de un referente que determina el margen de acción y la capacidad para articular y decidir sobre la marcha el manejo y el curso que deberán tomar las ideas musicales dentro de un discurso coherente.

Autores como Pérez (2010), Molina (2008), Nachmanovitch (2013), Nettl y Rusell (1988), entre otros, aseguran que la improvisación requiere de una preparación previa constituida sobre la base de las reglas y estructuras dentro de un género musical específico. La tarea creativa del improvisador consiste en trascender dichas reglas de forma novedosa durante la ejecución para lo cual debe contar, entre otros aspectos, con determinadas habilidades técnicas. Sin duda, dentro de la mayoría de los géneros, esta es una condición primordial que facilita la realización del discurso improvisado de manera solvente.

Evidentemente, hay diversas formas de improvisación y detrás de cada una de ellas hay determinados mecanismos que, con el paso del tiempo, se constituyen en un banco de insumos dispuestos para ser usados de forma anticipada. Cada improvisador 
construye su propio estilo porque es capaz de representar consigo mismo las exigencias de cada momento de manera creativa, prever las diferentes formas de llevar a cabo determinada tarea de improvisación, planificar una estrategia pertinente y controlar y regular las diversas condiciones y variables que surgen en las dinámicas creativas.

Para Berkowitz (2010), los elementos de la base de conocimientos deben ser organizados y refinados una vez instalados en la memoria, con el propósito de que el aprendiz tenga un acceso inmediato y creativo durante la improvisación. A partir de una profunda revisión de algunos sistemas de educación para la improvisación, el autor plantea cuatro estrategias fundamentales para su desarrollo: transporte, variación, recombinación y el uso de modelos que ejemplifiquen estos procesos en un contexto musical. Por ejemplo, aprender una fórmula musical en varias transposiciones o realizaciones variadas permite familiarizarse de una manera profunda con el material. Es posible incluso generar una red de asociaciones a partir de la recombinación de los diversos elementos musicales, lo cual permite crear diferentes configuraciones con resultados distintos y novedosos.

Además de la base de conocimientos, Pressing (1998) postula la idea del referente como un conjunto de circunstancias o condiciones propias del momento de la improvisación que originan y orientan la ejecución. Muchas de estas variables pueden ser predefinidas ensayadas y compartidas. El referente está encaminado, entre otros aspectos, a facilitar la toma de decisiones y permitir un desempeño con mayor énfasis en la creación.

El eje central y mediador por medio del cual se articulan la base de conocimientos, el referente y las prácticas para la interiorización de los materiales está constituido por el oído. Sin duda, es el oído el que cumple aquella función intermediaria y consciente entre mundo objetivo y subjetivo del músico improvisador. Tal y como lo plantea Mackamul (1982), "la audición interna es una imagen global que integra escritura, audición y ejecución, que sirve de base para captar, retener y reproducir conscientemente los sucesos musicales" (citado en Romero, 2013, s/p). Es un imperativo, como condición sine qua non, en el medio de los improvisadores, el desarrollo de la audición consciente que, de acuerdo con los postulados de Pressing, permite acrecentar la base de conocimientos.

Muchas veces, estas formas de actuación se dan a partir de conocimientos o 'saberes' prácticos que, como experiencias particulares, ha desarrollado de manera autónoma el improvisador y evolucionan de forma paulatina para explorar o resolver situaciones que suelen presentarse. De esta manera se conforma 'un estilo'; es decir, una manera propia de conjugar, de forma creativa, nuevas disposiciones musicales dentro del lenguaje respectivo. En consecuencia, la producción nunca es mecánica, por el contrario, refleja la personalidad del improvisador y evidencia su potencial imaginativo.

Estas experiencias facultan al improvisador tanto para prever situaciones y soluciones como para cambiar o mantener determinada propuesta creativa. En todo caso, debe saber leer o interpretar lo que puede pasar: el ambiente musical y las formas de intervención, entre otros aspectos. Claramente, esta forma de elaboración personal 
le permite no solo construir un estilo propio, sino desarrollar un pensamiento 'estratégico' porque posee un plan de acción previo para conducir su intervención, que dentro de determinados parámetros musicales da pie a la espontaneidad. El pensamiento estratégico del improvisador, entonces, incluye el juicio amparado en la experiencia para proponer y resolver determinadas intervenciones musicales tanto previsibles como imprevisibles.

Gainza (2002) sostiene que el improvisador utiliza "materiales sonoros y musicales" que obtiene del medio ambiente que lo rodea y de su "caudal musical internalizado" (p. 56). Durante el transcurso de la improvisación, el músico lleva a cabo una gama de procedimientos: expone, desarrolla, repite, re-edita, agrega o sustrae, por nombrar algunos. Al referirse al juego musical y a la improvisación, el autor asegura que ellos "contribuyen a la movilización y al metabolismo de las estructuras musicales internalizadas; al mismo tiempo, promueven la absorción de nuevos materiales y estructuras a través de la exploración y la manipulación creativa de los objetos sonoros" (p.56). Así se conforma una especie de juego a través del cual el músico crea espontáneamente su propia música.

Por otra parte, es imprescindible la reflexión concerniente al modo de trasmisión del saber improvisatorio, definido por las dinámicas de aprendizaje propias de los repertorios tradicionales. Evidentemente, la tradición oral es el proceso natural de enseñanza y resulta relevante extrapolar dos puntos de vista provenientes del campo de la filología que Pressing (1987) toma como referencia. En el primero, como procedimientos principales de transmisión de conocimiento, están la repetición y la imitación. Por lo general, esta explicación se apoya en la existencia de un supuesto urtext o texto original que con el tiempo sufre diversas transformaciones.

Un segundo modo de comprender las tradiciones orales, que desde el punto de vista de Pressing se adapta mejor a la improvisación, hace referencia al uso de fórmulas. A partir de esta teoría, Pressing sostiene que, así como los narradores (de tradición oral) cuentan con un repertorio de palabras, expresiones y condiciones de métrica con las que reelaboran las historias cada vez que las cuentan, los músicos improvisadores poseen un acervo de fórmulas, de temas y un método de composición:

$\mathrm{Al}$ elegir entre un repertorio de fórmulas de diferentes longitudes y, expandir o eliminar subtemas de acuerdo con las necesidades de la interpretación, el intérprete experimentado puede componer (en tiempo real, por lo tanto, improvisar) una versión detallada y fresca de una canción épica conocida" (Pressing, 1988, p. 14).

\section{Metodología}

La propuesta investigativa surgió desde una mirada cualitativa y el método a utilizar fue el estudio de caso. El propósito de este estudio de caso consistió en documentar una experiencia o evento en profundidad, o entender un fenómeno desde la 
perspectiva de quienes lo viven. Analizó a cada improvisador, en cuanto a sus motivaciones, experiencias, sus modos de operar, la forma como elige sus esquemas creativos, entre otros aspectos, que sirvieron para establecer las características comunes entre los improvisadores.

El estudio no partió de hipótesis ni de concepciones preestablecidas, sino que se generaron conforme se recolectaron y procesaron los datos. Aun cuando el conjunto de improvisadores compartiese algunos rasgos o formas de operación, cada uno de ellos vive condiciones distintas y posee una historia de vida diferente, por lo que su evaluación es única.

Frecuentemente, los estudios de caso de corte cualitativo se dirigen sobre la base de que pueden capturar el carácter único de una persona, situación o grupo, sin importar la generalización (Martin y Atkinson, 1994). Sin embargo, a partir de las consideraciones de Stake (2006), quien ofrece una tipología de estudios de casos que tiene que ver con el número de unidades o entidades a considerar, esta investigación centró su indagación en la yuxtaposición y contrastación de dichas unidades, en las cuales se pretendió revisar comparativamente similitudes y diferencias.

En esta indagación, el proceso para cada caso se 'repitió' en los demás. La revisión fue similar (se consideraron las mismas variables o aspectos, al igual que los instrumentos para recolectar los datos y el proceso en general, aunque puede haber algunos cambios). De cualquier forma, cada caso es un 'todo', una entidad por sí misma. Tanto la recolección de los datos como el análisis tuvieron como uno de sus objetivos explicar consistencias e inconsistencias entre cada uno de ellos. El nivel de análisis fue simultáneamente individual, caso por caso y colectivo, y para tal efecto se recurrió a las entrevistas y la observación participante entre el improvisador y el investigador, con el objetivo de documentar la experiencia o evento improvisatorio en profundidad o entender este hecho desde la perspectiva de quienes lo viven.

El estudio contó con el concurso de los siguientes improvisadores colombianos, reconocidos por ser figuras emblemáticas en este ámbito: Oscar Santafé (tiple), Camilo Andrés Cifuentes (requinto), Juan Miguel Sosa (guitarra), Ruth Marulanda (piano), Mauricio Murcia (clarinetista), Carlos Guzmán (guitarra), Joche Plata (percusión), Francis Lara (percusión) y Abelardo Jiménez (percusión).

El criterio de selección de estos artistas se realizó a través de un muestreo no probabilístico, con la intención de identificar aquellos músicos representativos que tuvieran estrecha relación con el núcleo temático, es decir, la improvisación en el marco de la música tradicional colombiana. Este muestreo funcionó a manera de una "bola de nieve" (Hernández, 2010), es decir que, una vez contactados los primeros improvisadores, ellos ofrecieron información relevante acerca de otros músicos con una experiencia reconocida en el contexto de la música andina y caribeña del país.

Las categorías de análisis que permitieron procesar la información dentro de la investigación fueron: a) Fundamentos del aprendizaje de la improvisación, que 
da cuenta de las condiciones del contexto formativo, en que el improvisador suele desarrollar sus habilidades de improvisación. b) El aprendizaje de la improvisación, que hace referencia a las diversas maneras en las cuales los improvisadores despliegan sus capacidades de improvisación, conservando el sentido del género en que realizan su actividad musical. c) Los insumos de la improvisación, que alude a los materiales con los que disponen los instrumentistas en su banco de memoria, como melotipos y ritmotipos, para cada género musical en particular.

\section{Resultados y análisis}

\subsection{Fundamentos del aprendizaje de la improvisación}

El papel de la improvisación en la música tradicional colombiana no es comparable con el que se tiene en el jazz, la música en la que por lo general se piensa cuando se habla de improvisación. En esos casos, las intervenciones improvisadas suelen presentarse como momentos de lucimiento solista, que generan un tipo de expectativas, análogos en sus dinámicas con el espectáculo virtuosista de la música europea académica. Por otro lado, como ya se ha dicho, la música de tradición se realiza con alto grado de espontaneidad y creatividad, directamente asociados a su función social y al entorno en el que surgen, pero centrados mayormente en procedimientos de variación desde los instrumentos acompañantes.

Como expresión creativa, la improvisación parte de un impulso personal moldeado por parámetros culturales. En el marco de este estudio, la música tradicional, hay que señalar que la fuerza que la origina no se encuentra solamente en la individualidad, sino que proviene de un acumulado social y un contexto estético concreto y delimitado.

El músico, en quien se centra este trabajo, desarrolla su oficio creativo desde dos vertientes. Una de ellas, el contexto social donde la actividad musical implica desempeñarse como improvisador, en una considerable parte de las ocasiones. Es frecuente hallar músicos populares que en las dinámicas culturales de su contexto se ven enfrentados a situaciones que requieren manejos y soluciones creativas.

Una segunda instancia propia de la tradición oral es la dinámica relacional maestroaprendiz, que favorece la formación improvisatoria de muchos instrumentistas. Allí encuentran, de primera mano, diversas estrategias de perfeccionamiento técnico y creativo. La experiencia directa, como principal modelo de aprendizaje, es moldeada por la intervención de un músico más avezado, quien enseña los procedimientos de creación y delimita el marco del estilo tradicional del género en cuestión. 
En palabras de Cifuentes (15 de agosto de 2016):

Para acercarse al estudio de la improvisación, hay que "untarse de pueblo", participar de las festividades y los espacios en donde se lleva a cabo esta práctica y sobre todo, aprender de los maestros, de aquellos que han improvisado a lo largo de la vida, quienes han elaborado suficientes criterios formativos para enseñar desde la propia tradición oral (comunicación personal).

Como parte de la tradición, y en su contexto social, cabe agregar que la improvisación es parte de un diálogo coherente entre el baile y su acompañamiento musical, lo cual obliga al improvisador a mantener una comunicación y atención constante con los bailarines. En el caso del torbellino en la música andina, dicho coloquio musical es enfatizado principalmente por el requintista, quien es el encargado de llevar la melodía principal y dirige, además, a los demás músicos. Es evidente la presencia de un diálogo entre él y su acompañamiento, como un medio para enriquecer los contrastes en la textura musical. "Los guitarristas de data tradicional, aprovechan los espacios que le otorga el requintista, para improvisar líneas melódicas muy bonitas en los bordones, y esto lo que hace es cambiar un poco y llenar más el tema" (Cifuentes, comunicación personal, 15 de agosto de 2016).

Una circunstancia similar se da en la costa Caribe, en el escenario de la cumbiamba, es decir, el lugar de la fiesta popular en que se baila la cumbia, donde el bailarín y el tambor alegre $^{1}$ se disputan la atención de la bailarina. Abelardo Jiménez cuenta cómo durante su primera experiencia en un festival como intérprete de este tambor, desconociendo la dinámica de su rol, vivió un momento de desconcierto cuando el bailarín "le puso la pata al tambor y lo tumbó" (Jimenez, comunicación personal, 15 de agosto de 2017). Esto sucedió en otra ocasión y, finalmente, gracias a la orientación de sus compañeros, comprendió que es parte del desarrollo de la coreografía, en la cual el cortejo implica que el bailarín intente desplazar al tamborero para conquistar a la bailarina.

En la costa Caribe, los gaiteros suelen emplear la expresión "tocar con asunto" para referirse a la importancia de establecer un diálogo musical entre los instrumentistas y entre ellos y la voz. Significa dotar a la improvisación de claridad y sentido, mediante el control de los matices de la obra en cuestión, sin interrumpir a los demás y, por lo general, mediante el esquema de introducción, desarrollo, clímax y final.

Con respecto al proceso específico de aprendizaje de la improvisación, Abelardo Jiménez (3 de agosto de 2017) asegura que el primer paso consiste en tomar como referencia a otros percusionistas de mayor trayectoria y con una gran experiencia como intérpretes del tambor alegre en formatos tradicionales. El procedimiento radica en escuchar e imitar a uno o varios percusionistas que por su particular modo de improvisación resulta importante y valioso emular y, de esta manera, comenzar a desarrollar un estilo propio (comunicación personal).

1 También llamado "tambor hembra", instrumento de percusión de origen africano usado en la costa Caribe colombiana. Su función consiste en proporcionar una línea básica a nivel rítmico en distintos aires tradicionales, como el bullerengue, la cumbia, la puya, entre otros. 
Este mismo tratamiento se encuentra en el centro del país referido a otros repertorios, pero con prácticas análogas. "La parte improvisatoria que he realizado es más de manera empírica y más por conocimiento de haber escuchado tanta música durante tantos años" (Murcia, comunicación personal, 19 de septiembre de 2015). Así, el aprendiz recoge de cada fuente algunas frases o motivos que pueden llegar a formar parte de su banco de insumos. En todo caso, observar, escuchar atentamente, indagar y reflexionar son los medios habituales para desarrollar la memoria y el oído interno como bases para la improvisación. Cifuentes (15 de agosto de 2016) asevera que:

Para uno aprender a improvisar, tiene que escuchar muchas personas que improvisen, y que uno pueda decir: esta frase está muy bacana, voy a hacer algo similar, no voy a hacer algo igual, pero voy a tomar elementos de allí para aplicarlos después en mi improvisación (comunicación personal).

Dicho recurso es fundamental, y el mecanismo que propone Cifuentes (15 de agosto de 2016) al respecto es el siguiente: "montar temas, y montarlos al oído, eso de sacar "partituritas" para el torbellino no es tan viable" (comunicación personal).

La dinámica de aprendizaje es un discurrir cíclico entre la imitación, el empleo de fórmulas y la creación misma. La imitación como punto de partida radica en la repetición con la intención expresa de memorizar y afianzar las habilidades propias de la ejecución instrumental y el material musical: una progresión armónica, el ritmo base, las frases melódicas, entre otros. Una vez realizado este proceso, la creación aparece cuando el improvisador está en condiciones de ubicar las ideas musicales adecuadas al género, la obra y el contexto.

Asegura el maestro Cifuentes, (comunicación personal, 15 de agosto de 2016) que la improvisación se basa en la recopilación de ideas musicales, ya sean propias o de otros, hecho que demanda del improvisador la capacidad tanto para seleccionar y memorizar como para hacer efectivas sus decisiones musicales. "Cualquier idioma que uno aprenda está aprendiendo por imitación. Solamente en la música nos damos el lujo de comenzar el lenguaje musical desde lo leído, eso es un exabrupto" (Romero, comunicación personal, 15 de octubre de 2016). Para aprender a improvisar es importante el contacto directo con la experiencia misma a través de la constante realimentación. "Es que el acercamiento, primero debe ser sobre el idioma, se tiene que acercar a la música; ¿cómo quiero enseñarle a leer primero? Primero lea y después le enseñó a hablar, esto está mal" (Cifuentes, comunicación personal, 15 de agosto de 2016).

A lo largo de todo el proceso de aprendizaje, un papel preponderante ha de cumplir la conciencia auditiva, reconocida en el medio musical, como el oído, que surge de la apreciación consciente de cada uno de los eventos sonoros y de las pautas para desarrollar los mecanismos de improvisación. Esta conciencia musical es una vía de descubrimiento que permite al improvisador elaborar, conectar y retener nuevos conocimientos y diversas formas de actuación. 
El oído puede considerarse como la cerradura del almacén donde están guardados todos los materiales musicales que pueden usarse al improvisar. Su función consiste en regular y direccionar aquello que está almacenado en la memoria, trayendo a la 'superficie' melodías, patrones, ritmos, entre otros elementos, que permitirán la creación de un discurso musical coherente.

El desarrollo adecuado de esta habilidad ofrece posibilidades como la de traducir lo escuchado directamente, sin la presencia o elaboración de operaciones mentales sucesivas. Un músico con buen entrenamiento auditivo desarrolla facilidad para tocar lo escuchado, recrea mentalmente cómo suena algo que lee, e incluso, cuando es el caso, asocia eficientemente los eventos sonoros a los signos de la grafía. Cuando se genera la correspondencia inmediata entre lo sonoro, lo escrito y la ejecución instrumental, se dice que 'se tiene oreja'.

\subsection{El aprendizaje de la improvisación}

La puesta en práctica de los materiales guarda estrecha relación con el ambiente en que se desarrolla. Al respecto, en el ámbito colombiano hay dos contextos claramente diferenciadores en el desarrollo de la práctica de improvisación. El primero de ellos, que es un modelo sui géneris, responde a un tipo de conocimiento organizado que surge de las visiones de tipo académico y, por otra parte, destaca el ámbito de aprendizaje en contexto social, basado en la experencia y la transmisión oral. En el primer caso, un ejemplo valioso lo constituye la experiencia académica del maestro Lácides Romero, cuya propuesta original gira en torno a contenidos que ha estructurado a lo largo de su carrera docente y es producto incluso de visiones contrarias a los paradigmas de la educación musical formal. Asegura Romero (30 de septiembre de 2015) que "el punto de partida no es la teoría... hay que comenzar haciendo praxis improvisatoria" (comunicación personal).

Los pilares de la estrategia metodológica en cuestión abarcan aspectos de menor a mayor complejidad a través del siguiente orden: improvisación gestual, rítmicolingüística, sobre escalas pentatónicas, en sistema blues, modal, armonía modal y, por último, la improvisación tonal. En palabras del maestro Romero, la capacidad creativa es inherente a todo ser humano, por tanto, más que añadir contenidos, el desafío consiste en desbloquear o quitar las barreras que imposibilitan la creación.

Dentro de la gran variedad de ejercicios, el siguiente ejemplo ilustra el caracter lúdico y la sencillez de su propuesta. 


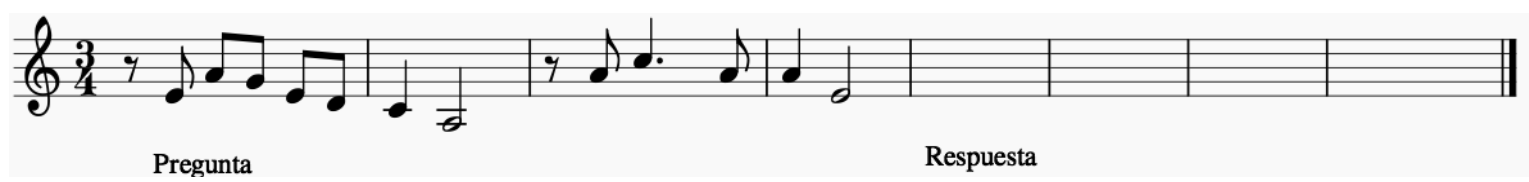

Figura 1. Modelo de ejercicio pregunta-respuesta propuesto por Lácides Romero Fuente: Romero (2016)

El segundo escenario reside en un sistema social en el que los improvisadores dentro del plano de la cotidianidad comparten de manera dialógica el uso de los materiales según las necesidades e intereses del momento. En este ambiente formativo, la gestión del conocimiento, es decir, el ejercicio de la creación espontánea, está dada entre el improvisador y el grupo, quienes comparten sus conocimientos de una forma recíproca. Esto es, una forma no-estructurada del conocimiento que está presente en las dinámicas prácticas de los saberes cotidianos de los improvisadores y es mantenida y producida colectivamente. En muchos casos, esta interacción está dirigida por un maestro o un músico con mayor experiencia encargado de transmitir los valores esenciales de la tradición.

En ambos contextos, el académico y el tradicional, el improvisador despliega la capacidad de tomar los diseños rítmicos y melódicos memorizados con anticipación y proporciona un orden lógico, con la clara opción de modificar de manera parcial o total este ordenamiento y manteniendo el sentido y el espíritu musical del género en cuestión.

Otro procedimiento consiste en tomar una melodía y crear variaciones en tiempo real, es decir, añadir o quitar notas, cambiar el ritmo o modificar en grados variables el diseño melódico. En este sentido, la inventiva del improvisador no consiste solamente en crear nueva música o nuevas frases, sino en disponer creativamente de las melodías que previamente registró en su memoria. Sossa (2016), argumenta:

Después de memorizar algunas melodías, lo que sigue es empezar a flexibilizarlas, digamos, súbala una octava, bájela una octava, transpórtela aquí re-armonice esto, después coja esta misma melodía que ya nos sabemos pero cámbiele unas notas cámbiele el ritmo varíele esto, solo utilice la primera parte y remátela de otra manera, invéntese el comienzo y llegue a la cola de la melodía que ya nos sabemos, puede ser incluso los pollitos, cambiémosle el modo a esos pollitos o lunita consentida lo que sea, cambiémosle el contorno, si subía ahora bajémoslo, busquemos otras notas del mismo acorde, tratemos de llegar ahora con saltos, y pues vamos inventando algunos ejercicios, digamos aproximaciones de tal tipo, y ahí viene lo teórico y ahí bien lo práctico, y asíllega el dominio (Sossa, comunicación personal, 15 de septiembre de 2016).

Murcia (2016), Sossa (2016), y Romero (2016), entre otros, confirman la tesis de Berkowitz (2010) y demás estudiosos de la improvisación con respecto al proceso que debe llevarse a cabo con los materiales que hacen parte de la base de conocimientos, 
los cuales deben ser reelaborados en múltiples versiones alrededor de parámetros rítmicos y melódicos. Todos están de acuerdo en que la relación entre la interiorización y la ejercitación continua hacen posible que los materiales surjan con facilidad y espontaneidad. De esta manera, la familiaridad y la práctica hacen que el evento improvisatorio se manifieste de forma natural.

\subsection{Los insumos de la improvisación}

Una condición básica para llegar a desarrollar las capacidades de improvisación consiste en adquirir previamente un buen dominio instrumental. Por ejemplo, el primer elemento en la base de conocimientos de los tamboreros, como condición previa para el desarrollo de habilidades improvisatorias, radica en el empleo de los diferentes 'golpes' a través de las diversas posturas de la mano al hacer contacto con el parche del tambor. ${ }^{2} \mathrm{Al}$ respecto, los tamboreros expertos otorgan especial importancia a la ejercitación constante de estos golpes antes de abordar la improvisación.

Con base en los patrones básicos y la práctica continua que fortalece su memoria de base, el improvisador lleva a cabo un juego de combinación de estos golpes y elabora frases de mayor complejidad que, en muchas ocasiones, suelen adoptarse como materiales propios. Paralelamente, el aprendiz debe desarrollar las habilidades musicales básicas para estas músicas, como controlar el pulso, cantar y bailar.

De acuerdo con Romero (2016) y Murcia (2016), el aprendizaje instrumental en cuanto al dominio técnico debe estar articulado con la apropiación de determinados materiales rítmicos o melódicos básicos que serán empleados posteriormente en la improvisación. Tal es el caso de los ejercicios de escalas y arpegios, para los instrumentos melódicos, que son practicados de un modo alejado de las formas tradicionales. Es posible combinar en un solo ejercicio varias tonalidades, por ejemplo, una escala ascendente en do mayor y descendente en fa sostenido, conectadas a través de una nota común o de aproximación. Así, el instrumentista desarrolla, por un lado, un abanico más amplio de habilidades motrices, y desarrolla flexibilidad para hacer uso de estos materiales dentro de la improvisación. Con esta finalidad, Romero propone adaptar ejercicios técnicos del piano como los de Hanon y Czerny a fórmulas rítmicas del repertorio colombiano.

No obstante, para llegar a la improvisación desde el dominio del instrumento es importante conocer a profundidad las estructuras rítmicas de cada género en particular. Asegura Marulanda (15 de septiembre de 2015) que es posible -y por gusto personal- acercarse al estudio de la improvisación bajo la condición de contar con las bases armónicas, melódicas y rítmicas a partir de los cuales podría llevarse a cabo cualquier realización dentro del ritmo del bambuco, pasillo, guabina o cualquier otro (comunicación personal). En otras palabras, es necesario saber

2 El dominio de estos golpes constituye el rudimento técnico de dicho instrumento, es decir, la adaptación de las condiciones físicas del ejecutante al uso del tambor. 
cómo es la conformación rítmica de cada género, determinado por la métrica y sus respectivas acentuaciones, así como la función rítmica de cada instrumento dentro de los formatos tradicionales.

Bajo este mismo principio, en el repertorio de la cumbia existen algunas combinaciones de motivos que son aprendidos como material común a todos los conocedores y que, por tanto, forman parte del lenguaje propio de cada aire musical. Algunos ejemplos al respecto, son los siguientes: tresillos a lo largo de dos compases, semicorcheas en dos o más pulsos de compás y combinaciones entre corcheas y semicorcheas durante dos o más compases.

En la tradición, como saber colectivo, para cada instrumento hay un banco de melotipos y ritmotipos disponibles para cada género. Es indispensable que el instrumentista memorice dichos contenidos para luego aplicarlos creativamente. En la zona andina del país se cuenta con una base de melotipos recurrentes que, a modo de fórmulas, suelen ser empleadas dentro del discurso musical. Al respecto, Sossa (15 de septiembre de 2016) asegura que, "los tocadores o improvisadores del torbellino más experimentados, son los que saben más frases y aprenden a unirlas, combinarlas y variarlas" (comunicación personal). Caso típico de estas fórmulas lo constituye el siguiente ejemplo que posee un contorno melódico y un patrón rítmico sencillos.

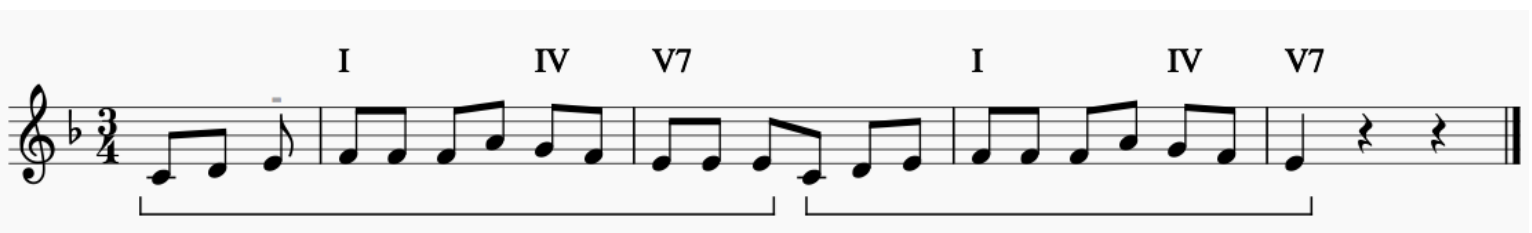

Figura 2. Ejemplo de melotipo característico del torbellino.

Fuente: Melo (2016).

La improvisación melódica en el torbellino a cargo del requinto, por ejemplo, funciona a partir de frases que deben coincidir con el marco armónico generalmente limitado a las funciones de tónica, subdominante y dominante. Estas frases melódicas poseen una duración corta, como si se tratara de un rompecabezas o un 'arma todo', ${ }^{3}$ y el improvisador intenta establecer la mayor coherencia posible combinando estas pequeñas melodías bajo su propio criterio. Algunos de los improvisadores denominan a este recurso como elasticidad melódica:

Improvisar es como coger unos bloques de arma todo, de Lego para ustedes, y armar diferentes cosas; pero los bloques están ahí, usted no puede inventarse otras figuras, en la bolsa ya hay unas. La creatividad está en cómo coges tus bloques y armas una estructura. Nunca se inventan otras figuras o fichas, lo que se hace es tomarlas y re disponerlas

3 Juego infantil conformado por bloques para ensamblar figuras y estructuras. 
creativamente para conseguir resultados moderadamente diferentes (Sossa, comunicación personal, 15 de septiembre de 2016)

En el contexto más tradicional de la música andina, el tiple cumple con la función de acompañante circunscrito, casi de manera exclusiva, al uso del sistema tonal. Dado que este diseño rítmico-armónico es estructural y estricto, el intérprete no puede modificarlo. Sin embargo, el componente rítmico ofrece posibilidades de variación determinadas por los cambios de acentuación y por los diferentes tipos de ataques, como se observa en los siguientes ejemplos:

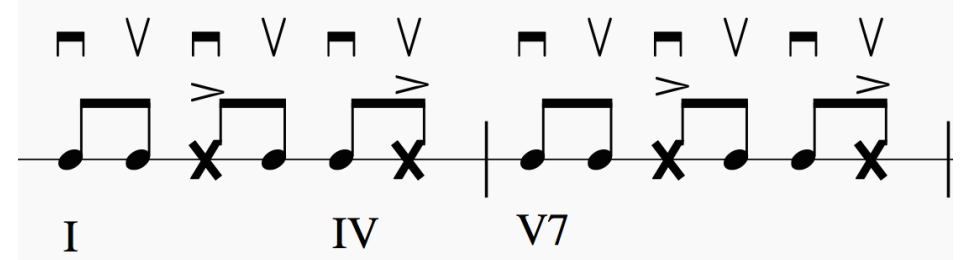

Figura 3. Patrón básico de acompañamiento del tiple.

Fuente: Melo (2016).

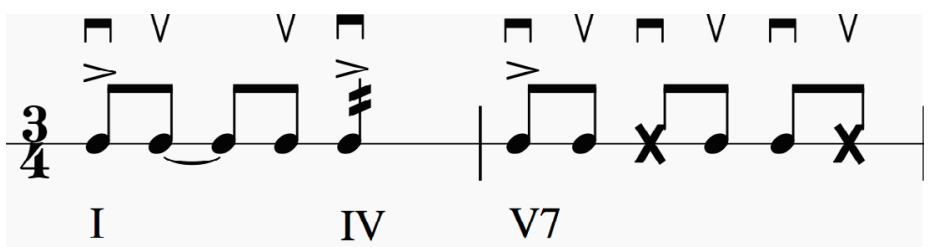

Figura 4. Variación rítmica sobre progresión armónica del torbellino.

Fuente: Melo (2016).

En la música tocada por conjuntos tipicos del centro del país, la guitarra tiene la función del bajo y complementa y enriquece la armonía. El rasgo creativo de la guitarra estriba en la realización de contra-melodías, que sirven para establecer un diálogo con el requinto y, a menudo, establecer conexiones entre acordes. En algunas ocasioens realiza pequeños solos improvisados durante las pausas de la melodía principal con el fin de dar un respiro al requinto. 


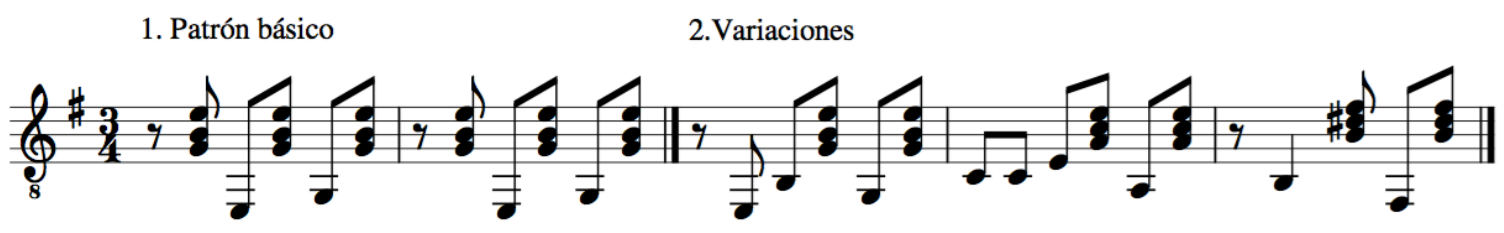

3. Bajos Improvisados
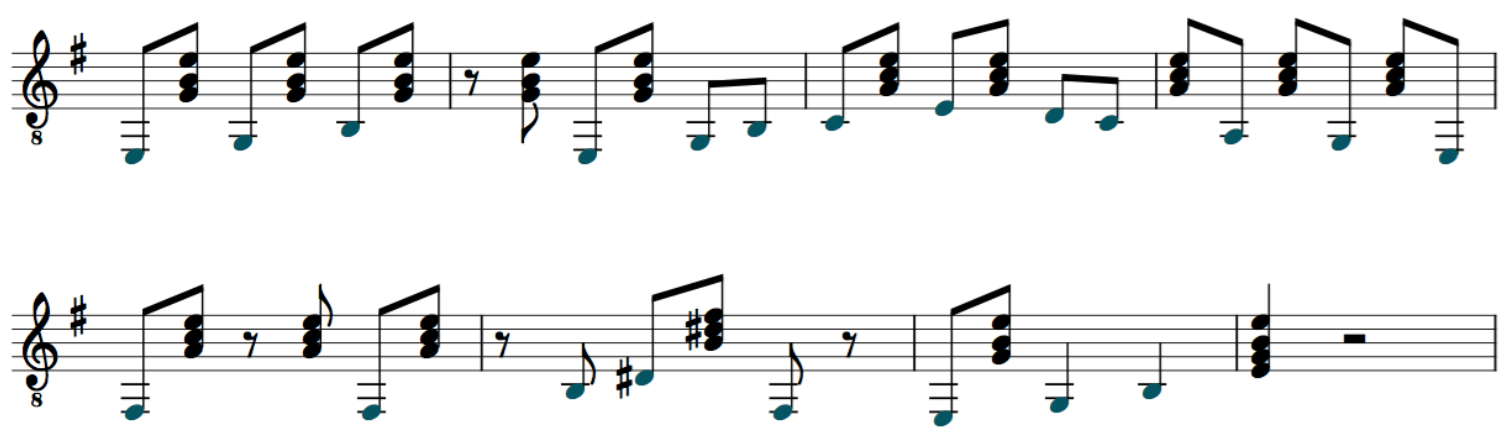

Figura 5. Formas de acompañamiento del bambuco en la guitarra.

Fuente: Guzmán (2016).

Asimismo, las versiones para piano de Ruth Marulanda presentan una gran elaboración textural originada en los mismos procesos, es decir, variaciones a los ritmos base, elaboraciones melódicas en el bajo y aprovechamiento de melotipos. Al respecto, asegura:

No sé, la verdad yo trato de agilizar las dos manos por igual, pero si, la mano izquierda, yo sí puedo ver la diferencia; cuando uno estudia normal la mano izquierda se dedica solamente a hacer armonías o sea a hacer acordes y acompañar. Yo ya me acostumbré a hacer melodías con la mano izquierda, entonces eso me sirvió mucho, por eso es que yo puedo jugar un poquito más. La gran mayoría de personas se limitan un poquito con la izquierda, a tocar acordes o acompañamientos. No, yo si le meto melodía, toda la que puedo (Marulanda, comunicación personal, 15 de septiembre de 2015).

A partir de sus versiones transcritas de piezas conocidas en el ámbito popular tradicional, formuladas como parte de un repertorio de iniciación para el piano; es posible contrastar con sus interpretaciones logradas en escena de forma espontanea. 


\section{La gata golosa}

Fulgencio García

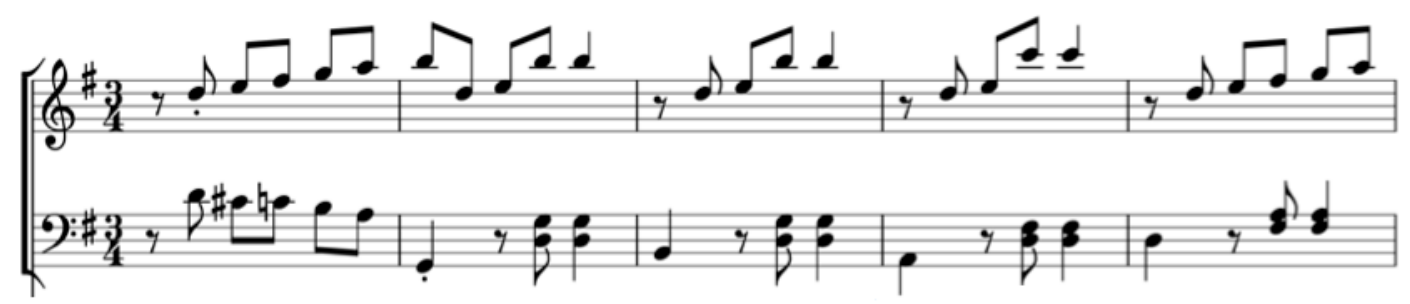

Versión de acompañamiento improvisada por Marulanda

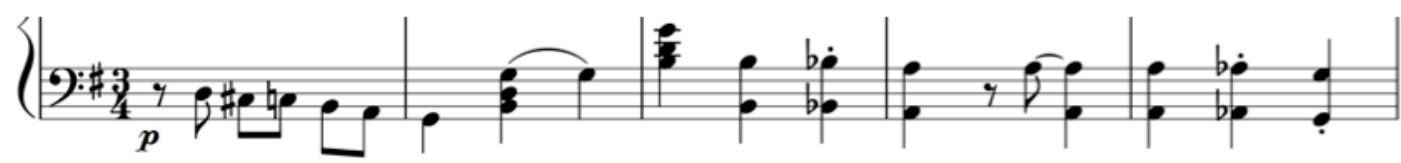

Figura 6. Versiones escritas e improvisadas.

Fuente: Marulanda (2016).

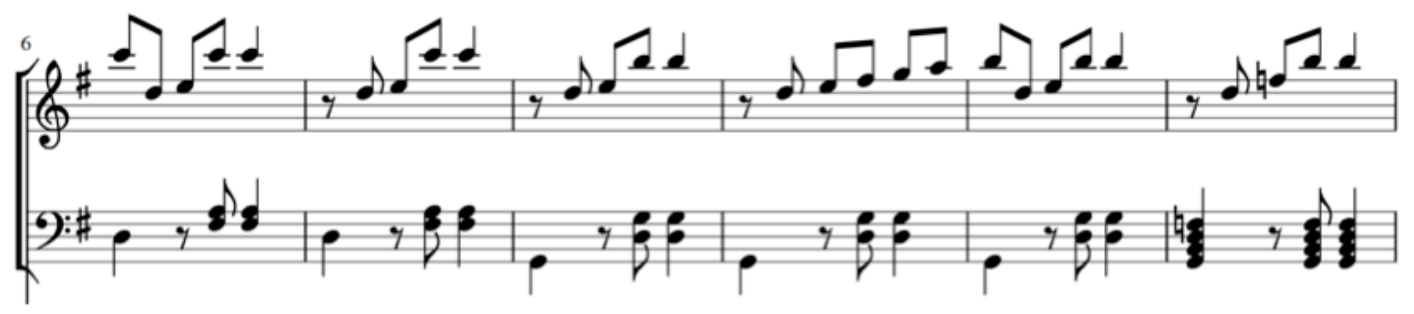

Versión de acompañamiento improvisada por Marulanda

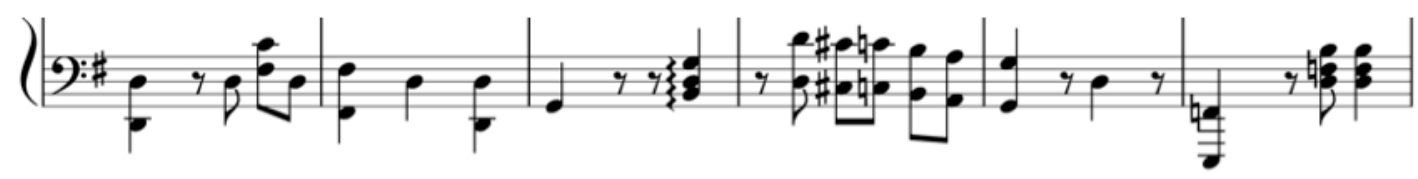

Figura 7. Versiones escritas e improvisadas.

Fuente: Marulanda (2016). 


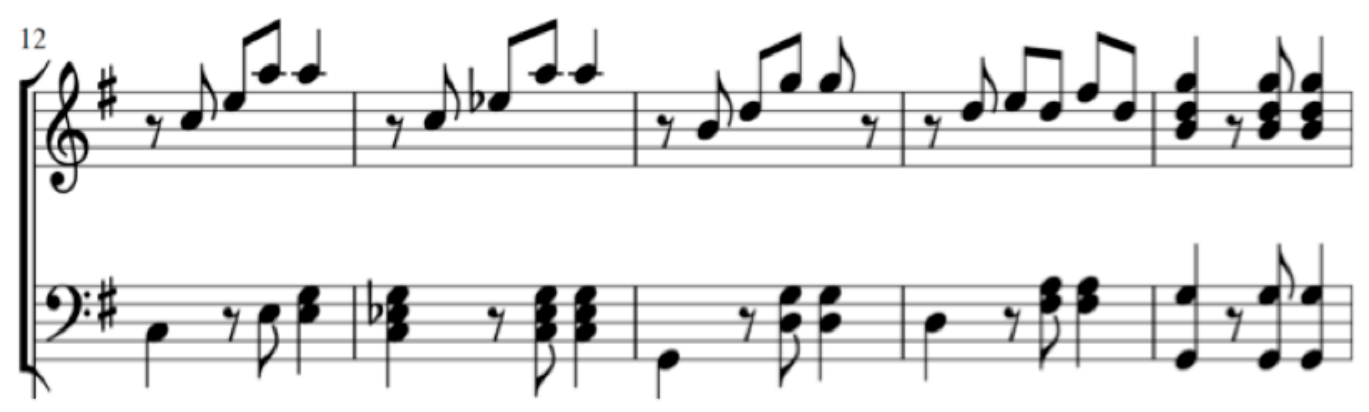

\section{Versión de acompañamiento improvisada por Marulanda}

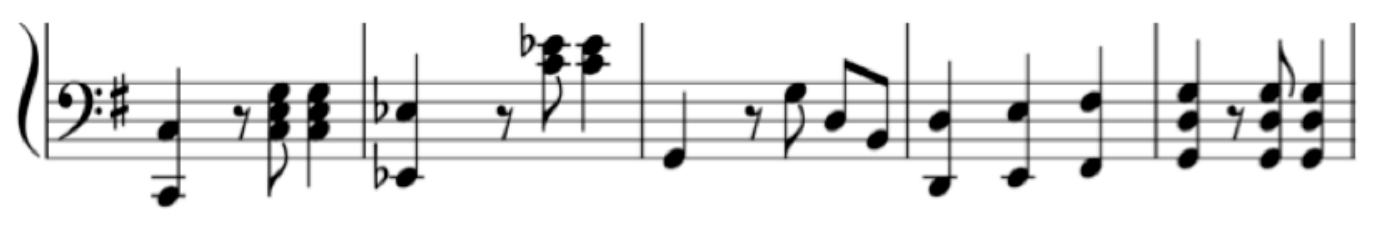

Figura 8. Versiones escritas e improvisadas.

Fuente: Marulanda (2016).

En el ejercicio de la tradición oral, los materiales, es decir, los melotipos, ritmotipos ${ }^{4}$ y todos los ejercicios mencionados, no se presentan de forma aislada con respecto al proceso de aprendizaje. Las unidades que hacen parte del juego improvisatorio suelen presentarse como un todo armónico. Sin embargo, esta no sería una base de conocimientos útil y flexible hasta tanto no se aplique en ejercicios improvisatorios.

\section{Conclusiones}

En concordancia con el propósito de identificar y comprender las prácticas de improvisación en contextos musicales, aparentemente disímiles como la costa Caribe colombiana y las músicas andinas del país, la investigación revela cómo los procesos de aprendizaje de la improvisación en estas dos regiones guardan entre sí elementos comunes y diferenciadores.

El ejercicio de la improvisación es un continuo dinámico entre lo individual y lo colectivo, hecho que exige disciplina y acatamiento a determinadas reglas musicales del contexto. Ejemplo de ello lo constituye el estudio individual en el toque de tambores

4 En esencia es una fórmula o diseño melódico o rítmico de uso frecuente en los contextos sociales musicales propios de una cultura que, a manera de 'plantilla', permite la creación de motivos o frases elaborados y combinados espontaneamente. 
en el marco de la cumbia o las realizaciones del requinto en el torbellino, hechos que pueden resultar insustanciales sin el concurso colectivo o la práctica con otros.

Un elemento común relevante lo constituye la relación entre la práctica de la improvisación y el desarrollo previo de habilidades técnicas para la ejecución instrumental: de manera insistente se resalta cómo el fomento de la primera favorece el desarrollo de la segunda.

Evidentemente, la formación instrumental de los improvisadores, así como su fundamentación teórica, no obedecen, en muchos casos, a formas ortodoxas de aprendizaje, sino que son obtenidos desde la praxis, la autoregulación y el interés de aprender constantemente. Inclusive, entre quienes han sido formados a nivel profesional, se registran casos de músicos prominentes que han desarrollado su capacidad creativa al margen de las reflexiones teóricas. Su formación como improvisadores se puede definir como ceñida a las estrategias empíricas. Como rasgo común en todos los casos, se observa que en dicho proceso es determinante la función de algunos referentes externos, ya sea directos como maestros y colegas, o indirectos como las fuentes encontradas en otros músicos, otras músicas, y otras experiencias de aprendizaje.

En general, también se encontró como un factor decisivo, presumiblemente el de mayor incidencia en la configuración de un perfil como improvisador, la capacidad auditiva, indispensable en la conformación de un banco de insumos y, en un nivel más avanzado, en la realización improvisada. Las operaciones llevadas a cabo a través de la habilidad auditiva son resaltadas desde todo punto de vista, tanto en la etapa de preparación para la improvisación como en la ejecución misma. Entendido como un grupo de funciones que relacionan los eventos sonoros con los conceptos y la grafía, el oído posibilita la inclusión de nuevo material a la memoria, permite valorar la precisión y adecuación del material creado e incluso, afirman los entrevistados, actúa como ente conductor en las creaciones espontaneas más elocuentes. Es citado por todos como un factor de la más alta trascendencia y, sin embargo, es notable que no haya luces sobre un método para el desarrollo de sus funcionalidades aplicadas a la improvisación.

La observación de las prácticas improvisatorias de la zona central - y en cierta medida las de la música costera- permite entender cómo sus realizaciones son reconocidas como fieles a la tradición cuando están apoyadas en el uso de fórmulas o materiales recurrentes. Su uso se ha extendido entre los intérpretes autóctonos y finalmente son aceptados como propios y tradicionales. En este estudio se han presentado algunos de estos ritmotipos y melotipos en función de su uso en el aprendizaje de la improvisación.

Ciertamente, la improvisación en la tradición musical colombiana cumple una función esencial, contraria a la idea que la define como una realización estática, rígida y terminada, que suele asociar lo tradicional con aquello que por definición no cambia. Desde esta perspectiva, la ' música de los viejos' se revela como una forma de expresión viva, cambiante, dinámica, que en cada ejecución se recrea a sí misma. 
Desde un enfoque práctico, es posible plantear la manera en que algunos aspectos importantes del proceso de preparación para la improvisación pueden recrearse en entornos académicos. Como punto de partida, por ejemplo, sería apropiado compilar el registro más completo posible de las formulas rítmico-melódicas correspondientes a cada género.

La presente investigación advierte que un modelo óptimo de aprendizaje de la improvisación será aquel que transite entre ambientes y, sobre todo, las prácticas propias de entornos empíricos y académicos. Los músicos improvisadores de esta indagación son un claro ejemplo de ello: sus experiencias y modos de aprender les han permitido plantear principios y metodologías con un orden estratégico y con aportes reflexivos valiosos alrededor de la improvisación. En tal evento, los procedimientos extraídos del ámbito académico pueden aportar recursos metodológicos y sus modelos de planificación de la enseñanza; a su vez, los músicos de la tradición oral están en condiciones de ofrecer sus pautas, recursos y formas metodológicas para direccionar el aprendizaje de la improvisación. Esto solo es posible en el momento en que los espacios de formación musical brinden opciones y oportunidades sistemáticas para acercarse y comprender las distintas maneras en que el músico de tradición oral lleva a cabo sus procesos creativos.

En últimas, la tarea consiste en articular el saber pedagógico formal con el saber del músico que hace parte de la tradición oral, en espacios de exploración y experimentación destinados al desarrollo de competencias en improvisación. 


\section{Referencias}

Bailey, D. (1992). Improvisation, its Nature and Practice in Music. New York: Da Capo Express.

Berkowitz, A. (2010). The Improvising Mind: Cognition and Creativity in the Musical Moment. New York: Oxford University Press.

Dalhaus, C. (1992). "Sur la determination du concept d'oeuvre musicale". Analyze Musicale, (29), 84-99.

Gainza, V. H de. (2002). Pedagogía musical. Dos décadas de pensamiento y acción educativa. Buenos Aires: Grupo Editorial Lumen.

Hernández, R. (2010). Metodología de la investigación. México, D. F.: McGraw Hill.

Mammersley, M. y Atkinson, P. (1994). Etnografía. Métodos de investigación (2.a ed). Buenos Aires: Paidós.

Melo, M. (2016). La improvisación en el torbellino desde las estrategias formativas de 3 intérpretes colombianos (Tesis de pregrado). Universidad Sergio Arboleda, Bogotá.

Molina, E. (1994). La improvisación aportaciones pedagógicas a la enseñanza musical. Recuperado de http://www.iem2.com/wp-content/uploads/2014/01/ Aportaciones.pdf

Nachmanovitch, S. (2013). Free Play: La improvisación en la vida y el arte. Buenos Aires: Paidós.

Nettl, B. y Russel, M. (2004). En el transcurso de la interpretación. Estudios sobre el mundo de la improvisación musical. Madrid: Ediciones Akal.

Pacheco, A. y Caraballo, R. (2011). La improvisación en el bombardino a partir del repertorio tradicional de las bandas del departamento de Córdoba: estudio preliminar para una guía de aprendizaje (Tesis de maestría). Universidad de Córdoba, Montería, Colombia. 
Pérez, J. (2010). “Un modelo teórico para el estudio de la improvisación en música”. Actas de la XVIII Jornadas de Jóvenes Investigadores de la AUGM. La investigación científica el eje del desarrollo regional en los albores del bicentenario de la independencia latinoamericana. Santa Fe: UNL Ediciones.

Pérez, J. y Montalvo, F. (2006). Improvisación en el pasillo y el bambuco de la región andina colombiana. Revista Contratiempo, (15). Recuperado de http://www. musigrafia.org/acontratiempo/?ediciones/revista15/artculos/improvisacin-en-elpasillo-y-el-bambuco-de-la-regin-andina-colombiana.html

Pressing, J. (1984). “Cognitive processes in improvisation”. En R. Chozier y A. Chapman (Eds.), Cognitive Processes in Perception of Art (pp. 345-363). Amsterdam: North-Holland.

Pressing, J. (1988). "Improvisation: methods and models". New York: Oxford University Press.

Rabinovitch, G. y Slominski, J. (2015). Towards a Galant Pedagogy: Partimenti and Schemata as Tools in the Pedagogy of Eighteenth-Century Style Improvisation. Music Theory Online, 21(3). Recuperado de https://scholarworks.gsu.edu/cgi/viewcontent. cgi?article=1056\&context=music_facpub

Romero, G. (2013). Oído interno, reflexiones sobre su definición, alcances y metodología de desarrollo. Recuperado de http://educacionauditivagermanromero. blogspot.com.co/2013/10/oído-interno-reflexiones-sobre-su.html

Sans, J. (s.f.). Patrones de improvisación y acompañamiento en la música venezolana de salón del siglo XIX. Revista Resonancias vol. 5, n8, mayo 2001, pp. 45-90. Escuela de Artes Universidad Central de Venezuela. Recuperado de http://resonancias.uc.cl/ es/N-8/patrones-de-improvisacion-y-acompanamiento-en-la-musica-venezolana-desalon-del-siglo-xix.html

Stake, R. (2006). Investigación con estudio de casos (2.a ed.). Madrid: Morata. 